\title{
Fenologia da floração, morfologia floral e sistema de incompatibilidade em espécies distílicas de Rubiaceae em fragmento florestal do Sudeste brasileiro $^{1}$
}

\author{
ZEFA V. PEREIRA ${ }^{2,4}$, MILENE F. VIEIRA $^{3}$ e RITA M. DE CARVALHO-OKANO ${ }^{3}$
}

(recebido: 14 de abril de 2005; aceito: 2 de agosto de 2006)

\begin{abstract}
Reproductive phenology, floral morphology and incompatibility system in distylous species of Rubiaceae in a forest fragment in southeastern Brazil). This work was carried out at the Estação de Pesquisa, Treinamento e Educação Ambiental Mata do Paraíso, Viçosa, Minas Gerais State, a forest fragment in the Atlantic Forest. Its main objective was to analyze the flowering phenology, floral morphology, and incompatibility system of the distylous species of Rubiaceae: Palicourea longepedunculata Gardner, P. marcgravii A. St.-Hil., Psychotria conjugens Müll. Arg., P. hastisepala Müll. Arg., P. hygrophiloides Benth., P. nuda (Cham. \& Schltdl.) Wawra, P. sessilis Vell. and Rudgea lanceolata Nutt. It also aimed to verify whether the floral morphs were in balanced equilibrium. The flowering in the species of Psychotria conjugens, $P$. hastisepala, P. hygrophiloides and $P$. sessilis were sequential, occurring during the rainy season (September through March). Significant differences were observed in the lengths of the stamens and styles between the thrum and pin flowers, except for $P$. hygrophiloides, in which only thrum flowers could be observed. Furthermore, dimorphism was observed in the length of stigmatic lobules, corollas, corolla lobules and anthers. Flowers opened in the morning lasting about $24 \mathrm{~h}$, except for $P$. nuda flowers, that lasted $48 \mathrm{~h}$. In most of the species the floral morph ratio was 1:1, except $P$. marcgravii, P. conjugens and P. hygrophiloides. Heteromorphic self-incompatibility was observed with growth inhibition of the incompatible pollen tubes occurring in the stigma of most of the species analyzed, except for the pin morphs of $P$. longepedunculata and $P$. hastisepala, in which reaction occurred in the style.
\end{abstract}

Key words - distyly, incompatibility system, Palicourea, Psychotria, Rudgea

RESUMO - (Fenologia da floração, morfologia floral e sistema de incompatibilidade em espécies distílicas de Rubiaceae em fragmento florestal do Sudeste brasileiro). O estudo foi conduzido na Estação de Pesquisa, Treinamento e Educação Ambiental Mata do Paraíso, localizada em Viçosa, Estado de Minas Gerais. Trata-se de fragmento florestal, inserido nos domínios de Floresta Atlântica. Objetivou-se analisar a fenologia da floração, a morfologia e biologia floral e o sistema de incompatibilidade de espécies distílicas de Rubiaceae: Palicourea longepedunculata Gardner, P. marcgravii A. St.-Hil., Psychotria conjugens Müll. Arg., P. hastisepala Müll. Arg., P. hygrophiloides Benth., P. nuda (Cham. \& Schltdl.) Wawra, P. sessilis Vell. and Rudgea lanceolata Nutt. Além disso, foi verificado se a razão entre os indivíduos dos dois morfos florais dessas espécies encontra-se em equilíbrio. As florações de Psychotria conjugens, P. hastisepala, P. hygrophiloides e P. sessilis foram seqüenciais e ocorreram durante a estação chuvosa (setembro a março). Foram registradas diferenças significativas entre alturas de estames e de estilete, entre flores brevistilas e longistilas, exceto em $P$. hygrophiloides; nessa espécie foram observadas apenas flores brevistilas. Além disso, constatou-se dimorfismo no comprimento dos lobos estigmáticos, da corola, dos lobos da corola e das anteras. As flores abriram-se pela manhã e duraram cerca de 24 horas, exceto as de $P$. nuda ( 48 horas). As espécies apresentaram razão equilibrada entre os morfos florais, exceto $P$. marcgravii, P. conjugens e $P$. hygrophiloides. Houve auto-incompatibilidade heteromórfica e a inibição do crescimento dos tubos polínicos incompatíveis ocorreu no estigma em todas as espécies analisadas, exceto nas formas longistilas de $P$. longepedunculata e $P$. hastisepala, nas quais a inibição dos tubos ocorreu no estilete.

Palavras-chave - distilia, Palicourea, Psychotria, sistema de incompatibilidade, Rudgea

\section{Introdução}

A família Rubiaceae possui cerca de 637 gêneros e 10.700 espécies (Robbrecht 1988), das quais 1.010 são observadas em território nacional (Barroso et al.

1. Parte da dissertação de mestrado da primeira autora, Programa de Pós-Graduação em Botânica da Universidade Federal de Viçosa, MG, Brasil

2. Doutoranda do Programa de Pós-Graduação da Universidade Estadual de Campinas, Departamento de Botânica, Caixa Postal 6109, 13083-970 Campinas, SP, Brasil.

3. Universidade Federal de Viçosa, Departamento de Biologia Vegetal, 36570-000 Viçosa, MG, Brasil.

4. Autor para correspondência: zefap@bol.com.br
1991). Representantes dessa família são importantes componentes de sub-bosques de florestas neotropicais (Gentry \& Emmons 1987), onde há alta diversidade de sistemas reprodutivos e de síndromes de polinização (Aizen \& Feinsinger 1994). Estudos sobre a biologia floral são, portanto, essenciais para o entendimento dessa diversidade e em espécies de Rubiaceae eles são insuficientes (Martin-Gajardo \& Morellato 2003).

As espécies de Rubiaceae apresentam diversas estratégias reprodutivas, sendo uma delas a distilia (Robbrecht 1988). Distilia é um dimorfismo floral, cujos morfos florais, brevistilos (B) e longistilos (L), produzidos 
em indivíduos distintos, diferem quanto à altura das estruturas reprodutivas, caracterizando, comumente, hercogamia recíproca (Ganders 1979, Dulberger 1992). Os morfos florais também podem diferir entre si, principalmente, em relação ao comprimento da corola, das anteras e dos lobos estigmáticos (Dulberger 1992, Herman et al. 1999). Além disso, a distilia caracteriza-se por apresentar mecanismo de incompatibilidade após polinizações ilegítimas (autopolinização e polinização intramorfo) e a produção de frutos é, geralmente, resultante de polinização legítima (polinização intermorfo, Barrett \& Richards 1990, Barrett 1992). O sucesso reprodutivo, contudo, depende de uma razão equilibrada (1:1) entre os morfos florais na população (Barrett 1992).

Após polinizações ilegítimas, ocorre, freqüentemente, reação de incompatibilidade, caracterizada pela interrupção do crescimento dos tubos polínicos no estigma (Nettancourt 1977). Entretanto, algumas espécies distílicas podem apresentar a reação de incompatibilidade na região mediana ou basal do estilete (Bawa \& Beach 1983, Faivre 2002, Rossi et al. 2005). Estudos que abordaram esse aspecto ainda são escassos (Bawa \& Beach 1983, Bawa et al. 1985, Gibbs 1990, Murray 1990, Ree 1997, Faivre \& McDade 2001), especialmente entre espécies brasileiras (Coelho \& Barbosa 2003, Castro e Araújo 2004, Castro et al. 2004, Teixeira \& Machado 2004a, Teixeira \& Machado 2004b, Consolaro et al. 2005, Rossi et al. 2005).

Os objetivos deste trabalho foram analisar a fenologia da floração, a morfologia e biologia floral e o local da reação de incompatibilidade, após polinizações ilegítimas, em flores de oito espécies distílicas de Rubiaceae, em remanescente de floresta estacional semidecidual, em Viçosa, Zona da Mata de Minas Gerais. Além disso, foi verificado se os morfos florais, das espécies estudadas, encontram-se em equilíbrio, na área de estudo.

\section{Material e métodos}

Local de estudo - O estudo foi realizado no período de junho de 2001 a setembro de 2002, na Estação de Pesquisa, Treinamento e Educação Ambiental Mata do Paraíso (EPTEAMP), onde foram registradas 30 espécies de Rubiaceae, 16 delas distílicas (Pereira et al. 2006). A EPTEAMP é fragmento com cerca de 194 ha, cujas coordenadas são $20^{\circ} 48^{\prime} 07^{\prime \prime} \mathrm{S}$ e $42^{\circ} 51^{\prime} 31^{\prime \prime}$ W. Sua vegetação natural faz parte dos domínios da Floresta Atlântica (Rizzini 1992) e, segundo a classificação de Veloso et al. (1991), caracteriza-se como floresta estacional semidecidual submontana. O clima de Viçosa, segundo a classificação de Köppen, é do tipo Cwa, ou seja, mesotérmico úmido, com verões quentes e chuvosos e invernos frios e secos (Vianello \& Alvez 1991). O total anual de precipitação, no período de estudo, foi de $970,26 \mathrm{~mm}$ e a temperatura de 20,3 ${ }^{\circ} \mathrm{C}$. Os meses de maior precipitação foram dezembro de 2001, janeiro e fevereiro de 2002 e os meses mais secos foram junho e julho de 2001 e abril e junho de 2002 (figura 1).

No presente estudo foram analisadas as seguintes espécies: Palicourea longepedunculata Gardner, Palicourea marcgravii A. St.-Hil., Psychotria conjugens Müll. Arg., Psychotria hastisepala Müll. Arg., Psychotria hygrophiloides Benth., Psychotria nuda (Cham. \& Schltdl.) Wawra, Psychotria sessilis Vell. e Rudgea lanceolata Nutt. Detalhes da morfologia e a taxonomia dessas espécies encontram-se no trabalho de Pereira et al. (2006). Material testemunho encontra-se depositado no acervo do Herbário VIC do Departamento de Biologia Vegetal da Universidade Federal de Viçosa, cujos registros, seguindo a ordem das espécies apresentadas anteriormente, são: 26953, 26956, 26963, 26964, $26807,26679,26970$ e 26959.

Fenologia da floração, morfologia e biologia floral - O estudo fenológico foi realizado em quatro espécies por serem abundantes e ocorrerem próximas entre si. Para tanto, foram etiquetados 20 indivíduos de Psychotria conjugens, 30 de $P$. hastisepala, 40 de $P$. hygrophiloides e 52 de $P$. sessilis, os quais foram acompanhados semanalmente a fim de se verificar a presença ou ausência de botões florais e/ou flores.

A análise morfológica foi realizada em todas as espécies estudadas. Para cada espécie, foram coletadas 10 flores de cada morfo floral, das quais foram tomadas com auxílio de um paquímetro (erro $0,05 \mathrm{~mm}$ ), as seguintes medidas: altura do estilete e dos estames, comprimento da corola, dos lobos da corola, dos lobos estigmáticos e das anteras. As medidas dos morfos florais, de cada espécie, foram comparadas utilizandose o teste $t$ (Zar 1999), exceto em P. hygrophiloides, que

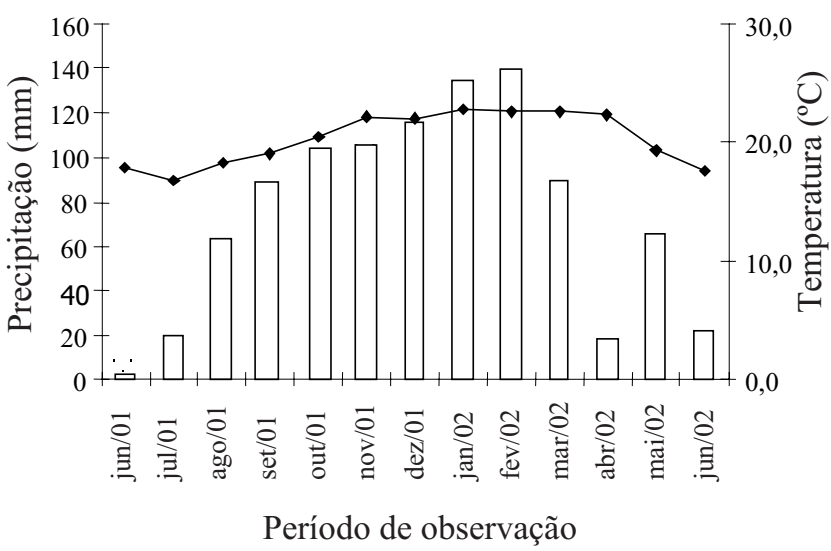

Figura 1. Precipitação mensal ( $\square$ ) e temperatura média mensal (-), no período de junho de 2001 a junho de 2002, em Viçosa, Minas Gerais.

Figure 1. Monthly rainfall ( $\square$ ) and temperature (-), June 2001 through June 2002, in Viçosa, Minas Gerais. 
apresentou apenas um dos morfos florais.

O período de antese e a duração das flores foram observados em 20 flores de cada morfo floral, provenientes de oito indivíduos diferentes, em todas as espécies estudas, exceto em $P$. longepedunculata (seis indivíduos).

Razão entre morfos florais e sistema de incompatibilidade - Para verificar a razão entre os morfos florais das espécies estudadas, foi identificado o tipo floral de todos os indivíduos observados em floração, ao longo de uma trilha de $1.400 \mathrm{~m}$ de comprimento por $10 \mathrm{~m}$ de largura.

O sistema de incompatibilidade foi analisado em Palicourea longepedunculata, Psychotria hastisepala, P. hygrophiloides, P. nuda, P. sessilis e Rudgea lanceolata utilizando-se a metodologia de Bawa \& Beach (1983), ou seja, realizando-se as seguintes polinizações manuais: $\mathrm{B} \times \mathrm{B}$ e $\mathrm{L} \times$ $\mathrm{L}$ (autopolinizações); $\mathrm{B} \times \mathrm{B}$ e $\mathrm{L} \times \mathrm{L}$ (intramorfos); $\mathrm{B} \times \mathrm{L}$ e $\mathrm{L} \times \mathrm{B}$ (intermorfos). Em P. hygrophiloides foram realizados apenas polinizações ilegítimas, em flores brevistilas. Foram utilizadas 24 flores por tratamento, totalizando 144 flores analisadas por espécie. Em todas as espécies, exceto P. hastisepala, as polinizações foram realizadas in vitro. Foram coletados ramos com botões, em pré-antese, que foram mantidos em água. Na antese, as flores foram removidas dos ramos, polinizadas e mantidas em caixas plásticas (gerbox) contendo gelatina natural, por um período de 12, 18 e 24 horas, exceto $P$. nuda, cujo período foi de 30,36 e 48 horas, pois suas flores duravam mais que 24 horas. Após esses períodos, as flores foram estocadas em etanol 70\%. Em P. hastisepala, as polinizações foram feitas in vivo, uma vez que suas flores murchavam quando os ramos eram removidos da planta. Para tanto, botões em pré-antese foram emasculados e isolados em sacos de organza e os tratamentos foram realizados no dia seguinte. Após 12, 18 e 24 horas, as flores foram coletadas e estocadas em etanol 70\%. O pistilo de cada flor foi clareado com $\mathrm{NaOH}(9 \mathrm{~N})$ e colorido com azul de anilina solução aquosa, para observação do crescimento de tubos polínicos, em microscopia de fluorescência (Martin 1959).

\section{Resultados}

Fenologia da floração, morfologia e biologia floral - A floração das espécies estudadas ocorreu na estação chuvosa (setembro a março), período que coincide com os meses mais quentes do ano (figura 1). As florações foram seqüenciais, iniciadas por $P$. sessilis, seguida por $P$. hygrophiloides, $P$. conjugens e, por último, P. hastisepala, havendo sobreposição do período de floração entre espécies (figura 2).

$\mathrm{Na}$ tabela 1 e figura 4 encontram-se as diferenças entre altura dos estames e do estilete em flores brevistilas e longistilas, das espécies estudadas. P. hygrophiloides apresentou somente flores brevistilas (tabela 1). Pequena sobreposição na altura dos estames e do estilete foi registrada em $P$. hastisepala (figura 3); apesar disso, também foi registrada diferença significativa entre os

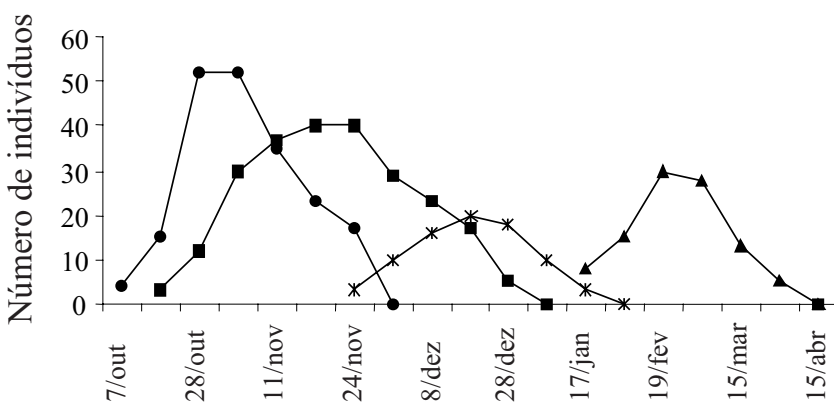

Datas de observação

Figura 2. Número de indivíduos de Psychotria sessilis Vell. (--), P. hygrophiloides Benth. (-口-), P. conjugens Müll. Arg. $\left(-\star_{-}\right)$e P. hastisepala Müll. Arg. $(-\mathbf{\Lambda}-)$ com botões florais e/ou flores, no período de outubro de 2001 a abril de 2002, Viçosa, Minas Gerais.

Figure 2. Number of individuals of Psychotria sessilis Vell. (--), P. hygrophiloides Benth. (--), P. conjugens Müll. Arg. (-丸-) e P. hastisepala Müll. Arg. (- - -) with flower buds and /or flowers, October 2001 through April 2002, in Viçosa, Minas Gerais.

seus morfos florais (tabela 1).

O comprimento dos lobos estigmáticos foi significativamente diferente entre os morfos florais de todas as espécies (tabela 1). O comprimento da corola diferiu significativamente em $P$. longepedunculata, P. marcgravii, $P$. hastisepala, $P$. nuda e $R$. lanceolata (tabela 1). O comprimento das anteras diferiu significativamente em $P$. marcgravii e $P$. nuda (tabela 1). O comprimento dos lobos da corola diferiu significativamente em $P$. longepedunculata (tabela 1). As flores brevistilas mostraram-se maiores que as longistilas em todas essas medições.

Em todas as espécies observadas, as flores abriramse pela manhã e duraram cerca de 24 horas, exceto em P. nuda, cujas flores duraram cerca de 48 horas.

Razão entre morfos florais e sistema de incompatibilidade - A proporção de indivíduos com flores brevistilas e longistilas variou nas espécies estudadas (tabela 2). P. marcgravii e P. conjugens apresentaram proporção de cerca de 2:1 e P. hygrophiloides de 1:0; nas demais espécies, os morfos florais encontravam-se em proporção equilibrada.

Em todos os pistilos, nas polinizações legítimas, os tubos polínicos cresceram até o ovário e alcançaram os óvulos, após 24 horas, em $P$. longepedunculata, $P$. hastisepala, $P$. sessilis e $R$. lanceolata e, após 36 horas, 
Tabela 1. Média \pm Desvio Padrão de medidas realizadas em flores distílicas de Rubiaceae, em Viçosa, Minas Gerais: $(\mathrm{Pl}=$ Palicourea longepedunculata Gardner; $\mathrm{Pm}=$ P. marcgravii A. St.-Hil.; Pc = Psychotria conjugens Müll. Arg.; Ph = P. hastisepala Müll. Arg.; Phy = P. hygrophiloides Benth.; Pn = P. nuda (Cham. \& Schltdl.) Wawra; Ps = P. sessilis Vell.; Rl = Rudgea lanceolata Nutt. B = flor brevistila; $\mathrm{L}=$ flor longistila).

Table 1. Mean \pm Standard Deviation of measurements taken in distylous flowers of Rubiaceae, in Viçosa, Minas Gerais: $(\mathrm{Pl}=$ Palicourea longepedunculata Gardner; $\mathrm{Pm}=$ P. marcgravii A. St.-Hil.; Pc = Psychotria conjugens $\mathrm{Müll}$. Arg.; Ph = P. hastisepala Müll. Arg.; Phy= P. hygrophiloides Benth.; Pn = P. nuda (Cham. \& Schltdl.) Wawra; Ps = P. sessilis Vell.; Rl=Rudgea lanceolata Nutt. $\mathrm{B}=$ thrum ; $\mathrm{L}=$ pin).

\begin{tabular}{|c|c|c|c|c|c|c|c|}
\hline \multirow{3}{*}{$\begin{array}{l}\text { Espécie } \\
\text { primento }\end{array}$} & \multirow{3}{*}{$\begin{array}{l}\text { Flor e } \\
\text { teste } t\end{array}$} & \multicolumn{2}{|c|}{ Corola } & \multicolumn{2}{|c|}{ Estilete } & \multicolumn{2}{|c|}{ Estames } \\
\hline & & Comprimento & Comprimento & Altura & Comprimento & Altura & $\mathrm{C}$ o m- \\
\hline & & $\begin{array}{l}\text { dos lobos } \\
\quad(\mathrm{mm})\end{array}$ & $(\mathrm{mm})$ & $(\mathrm{mm})$ & $\begin{array}{c}\text { dos lobos estigmáticos } \\
(\mathrm{mm})\end{array}$ & $(\mathrm{mm})$ & $\begin{array}{l}\text { das anteras } \\
(\mathrm{mm})\end{array}$ \\
\hline \multirow[t]{3}{*}{$\overline{\mathrm{Pl}}$} & $\bar{B}$ & $11,75 \pm 1,23$ & $2,28 \pm 0,51$ & $7,50 \pm 0,82$ & $2,07 \pm 0,12$ & $9,75 \pm 0,98$ & $2,21 \pm 0,40$ \\
\hline & $\mathrm{L}$ & $8,27 \pm 0,79$ & $1,57 \pm 0,40$ & $9,15 \pm 1,00$ & $0,44 \pm 0,10$ & $6,05 \pm 0,73$ & $2,21 \pm 0,24$ \\
\hline & $\mathrm{t}$ & $7,54 *$ & $3,466 * *$ & $-4,04 *$ & $34,15 *$ & $11,80 *$ & $1,00 \mathrm{~ns}$ \\
\hline \multirow[t]{3}{*}{ Pm } & B & $21,85 \pm 1,33$ & $3,25 \pm 0,64$ & $11,65 \pm 1,86$ & $2,90 \pm 0,31$ & $19,95 \pm 1,77$ & $4,98 \pm 0,43$ \\
\hline & $\mathrm{L}$ & $18,70 \pm 1,16$ & $3,65 \pm 0,63$ & $20,60 \pm 1,07$ & $0,14 \pm 0,08$ & $13,40 \pm 0,52$ & $3,50 \pm 0,47$ \\
\hline & $\mathrm{t}$ & $5,64 *$ & $-1,42 \mathrm{~ns}$ & $-13,19 *$ & $26,67 *$ & $11,23 *$ & $7,36 *$ \\
\hline \multirow[t]{3}{*}{$\mathrm{Pc}$} & $\mathrm{B}$ & $7,15 \pm 0,67$ & $2,00 \pm 0,24$ & $4,20 \pm 0,42$ & $1,63 \pm 0,26$ & $7,02 \pm 0,64$ & $1,95 \pm 0,16$ \\
\hline & $\mathrm{L}$ & $6,75 \pm 0,79$ & $2,00 \pm 0,34$ & $7,30 \pm 0,42$ & $0,45 \pm 0,15$ & $4,70 \pm 0,63$ & $1,96 \pm 0,08$ \\
\hline & $\mathrm{t}$ & $1,22 \mathrm{~ns}$ & $0,00 \mathrm{~ns}$ & $-16,44 *$ & $12,47 *$ & $8,17 *$ & $-0,176 \mathrm{~ns}$ \\
\hline \multirow[t]{3}{*}{$\mathrm{Ph}$} & B & $14,18 \pm 1,37$ & $2,72 \pm 0,36$ & $8,57 \pm 0,52$ & $2,75 \pm 0,30$ & $12,87 \pm 1,21$ & $2,86 \pm 0,21$ \\
\hline & $\mathrm{L}$ & $11,60 \pm 1,27$ & $2,15 \pm 0,47$ & $10,80 \pm 1,47$ & $0,96 \pm 0,08$ & $9,99 \pm 1,47$ & $2,41 \pm 0,56$ \\
\hline & $\mathrm{t}$ & $4,80 *$ & 3,04 ns & $-4,51 *$ & $18,22 *$ & $6,28 *$ & $2,37 \mathrm{~ns}$ \\
\hline \multirow[t]{3}{*}{ Phy } & $\mathrm{B}$ & $6,20 \pm 0,43$ & $1,42 \pm 0,31$ & $4,04 \pm 0,42$ & $1,14 \pm 0,18$ & $6,30 \pm 0,43$ & $1,13 \pm 0,17$ \\
\hline & $\mathrm{L}$ & - & - & - & - & - & - \\
\hline & $\mathrm{t}$ & - & - & - & - & - & - \\
\hline \multirow[t]{3}{*}{$\mathrm{Pn}$} & B & $22,90 \pm 1,29$ & $5,05 \pm 0,90$ & $9,35 \pm 0,41$ & $2,79 \pm 0,27$ & $19,40 \pm 1,17$ & $5,40 \pm 0,47$ \\
\hline & $\mathrm{L}$ & $21,00 \pm 1,83$ & $5,67 \pm 0,57$ & $20,08 \pm 0,58$ & $1,32 \pm 0,16$ & $13,00 \pm 1,05$ & $4,43 \pm 0,49$ \\
\hline & $\mathrm{t}$ & $2,69 *$ & $1,87 \mathrm{~ns}$ & $-47,98 *$ & $14,66 *$ & $12,84 *$ & $4,64 *$ \\
\hline \multirow[t]{3}{*}{ Ps } & B & $7,75 \pm 0,54$ & $3,73 \pm 0,53$ & $5,05 \pm 0,90$ & $1,70 \pm 0,54$ & $7,85 \pm 0,47$ & $1,40 \pm 0,29$ \\
\hline & $\mathrm{L}$ & $7,60 \pm 0,52$ & $3,50 \pm 1,00$ & $7,80 \pm 1,11$ & $0,91 \pm 0,12$ & $5,55 \pm 0,50$ & $1,31 \pm 0,31$ \\
\hline & $\mathrm{t}$ & $0,64 \mathrm{~ns}$ & $0,64 \mathrm{~ns}$ & $-6,09 *$ & $4,54 *$ & $10,58 *$ & $0,60 \mathrm{~ns}$ \\
\hline \multirow[t]{3}{*}{ Rl } & $\mathrm{B}$ & $9,95 \pm 1,50$ & $2,15 \pm 0,16$ & $5,15 \pm 0,34$ & $1,78 \pm 0,31$ & $8,30 \pm 1,08$ & $1,06 \pm 0,10$ \\
\hline & $\mathrm{L}$ & $7,95 \pm 0,69$ & $2,45 \pm 0,50$ & $10,35 \pm 1,00$ & $0,79 \pm 0,18$ & $4,95 \pm 0,60$ & $1,00 \pm 0,09$ \\
\hline & $\mathrm{t}$ & $3,84 *$ & $-1,82 \mathrm{~ns}$ & $-15,56 *$ & $8,86 *$ & $8,70 *$ & $1,41 \mathrm{~ns}$ \\
\hline
\end{tabular}

${ }^{*} P<0,01 ; * * P<0,05 ; \mathrm{ns}=$ diferenças não-significativas. ${ }^{*} P<0,01 ; * * P<0,05 ; \mathrm{ns}=$ non-significant differences.

em P. nuda. Em P. hygrophiloides, a ausência de um dos morfos florais impossibilitou verificar o crescimento dos tubos até o ovário. Nas polinizações ilegítimas, houve inibição do crescimento de tubos polínicos (figuras 45) em todos os 24 pistilos observados, caracterizando a incompatibilidade heteromórfica. Nas flores brevistilas, de todas as espécies, a inibição de tubos polínicos ocorreu no estigma (figura 4), assim como nas longistilas, exceto em $P$. longepedunculata (figura 5) e $P$. hastisepala. Nessas espécies, os tubos polínicos cresceram em mais da metade do estilete (figuras 6-7).

\section{Discussão}

A floração das espécies estudadas é do tipo "anual" (sensu Newstron et al. 1994). De acordo com esses autores, a duração da floração por um a cinco meses, tal como observado nas espécies estudadas, é denominada de "anual-intermediária". Esse mesmo padrão sazonal foi observado por Almeida \& Alves (2000) e MartinGajardo \& Morellato (2003) em espécies de Rubiaceae, na Floresta Atlântica. Almeida \& Alves (2000) verificaram que a floração de Psychotria nuda e de $P$. brasiliensis Vell. ocorreram na estação seca, na Ilha Grande, Rio de Janeiro. Por outro lado, Martin-Gajardo \& Morellato (2003) observaram que o máximo de espécies de Rubiaceae (70\% das 10 espécies estudadas), com flores em antese, ocorreu na estação chuvosa, em Ubatuba, São Paulo, semelhante ao registrado no presente estudo. Costa et al. (1997) mencionaram que, 

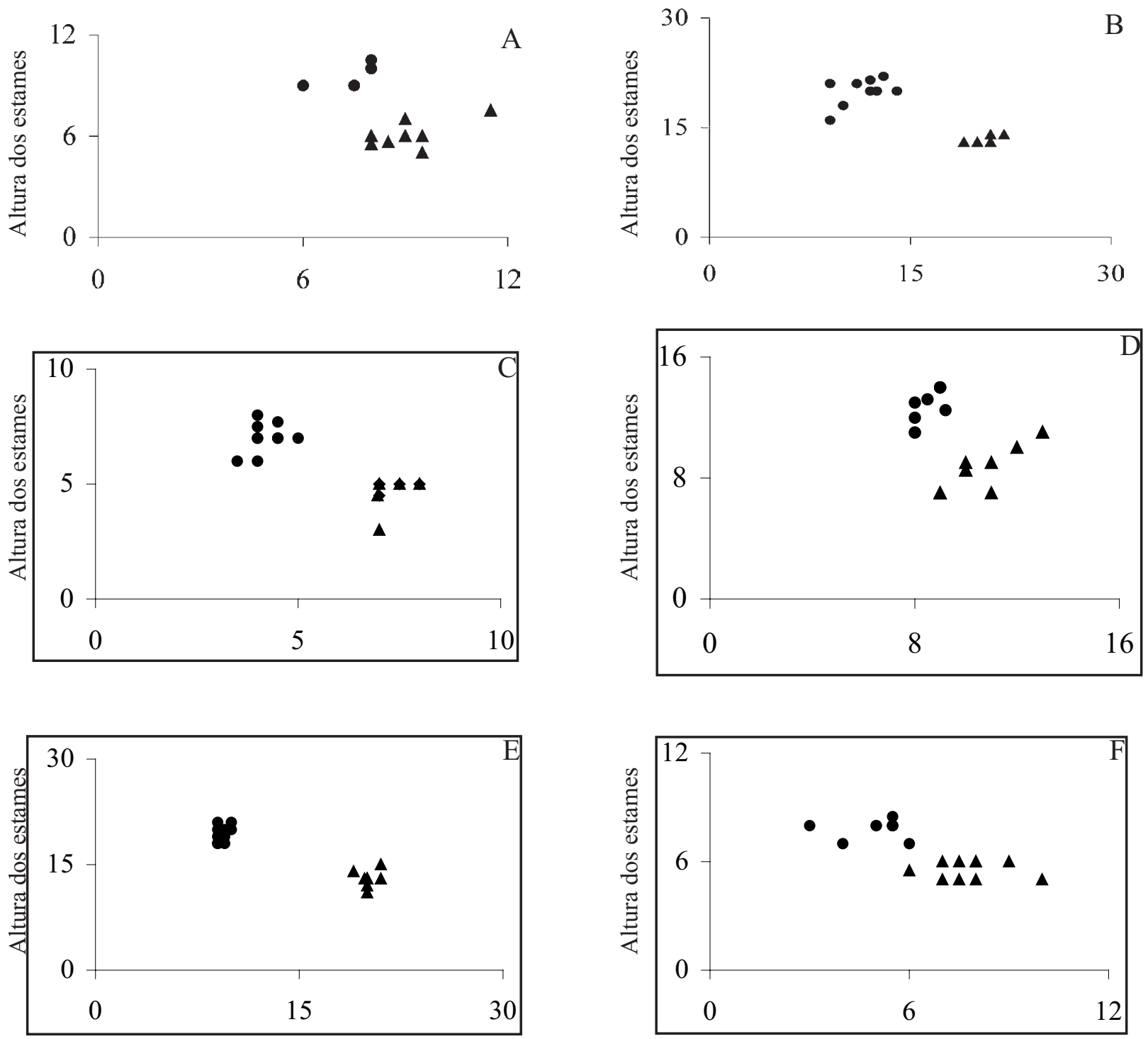

Altura do estilete

Altura do estilete

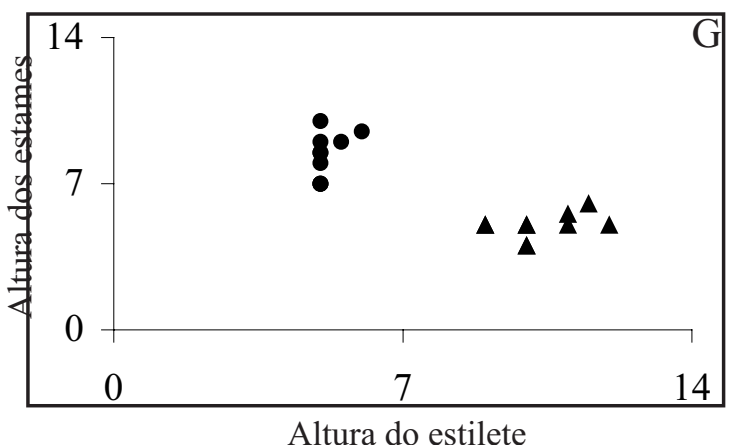

Figura 3. Altura (mm) dos estames e dos estiletes de flores brevistilas $(\bullet)$ e longistilas $(\mathbf{\Delta})$ de espécies distílicas de Rubiaceae, em Viçosa, Minas Gerais. A. Palicourea longepedunculata Gardner. B. P. marcgravii A. St.-Hil. C. Psychotria conjugens Müll. Arg. D. P. hastisepala Müll. Arg. E. P. nuda (Cham. \& Schltdl.) Wawra. F. P. sessilis Vell. G. Rudgea lanceolata Nutt.

Figure 3. Height $(\mathrm{mm})$ of the stamens and styles in thrum flowers $(\bullet)$ and pin flowers $(\mathbf{\Delta})$ of the distylous species of Rubiaceae, in Viçosa, Minas Gerais. A. Palicourea longepedunculata Gardner. B. P. marcgravii A. St.-Hil. C. Psychotria conjugens Müll. Arg. D. P. hastisepala Müll. Arg. E. P. nuda (Cham. \& Schltdl.) Wawra. F. P. sessilis Vell. G. Rudgea lanceolata Nutt. 
Tabela 2. Proporção de indivíduos com flores brevistilas e longistilas, de espécies distílicas de Rubiaceae em Viçosa, Minas Gerais.

Table 2. Proportion of specimens with thrum and pin flowers of distylous species of Rubiaceae, in Viçosa, Minas Gerais.

\begin{tabular}{lccc}
\hline \multirow{2}{*}{ Espécie } & \multicolumn{2}{c}{ Morfo floral ( $\mathrm{n}^{\mathbf{0}}$. de indivíduos) } & Proporção \\
\cline { 2 - 3 } & Brevistila & Longistila & $1: 1$ \\
\hline Palicourea longepedunculata & 3 & 3 & $2: 1$ \\
P. marcgravii & 6 & 3 & $2: 1$ \\
Psychotria conjugens & 16 & 8 & $1: 1$ \\
P. hastisepala & 16 & 15 & $1: 0$ \\
P. hygrophiloides & 45 & 0 & $1: 1$ \\
P. nuda & 5 & 6 & $1: 1$ \\
P. sessilis & 25 & 12 & $1: 1$ \\
Rudgea lanceolata & 10 & 12 & \\
\hline
\end{tabular}

além dos fatores abióticos, também devem ser levados em consideração os caracteres genéticos das plantas e a sua interação com os polinizadores, dispersores e predadores de semente, como fatores reguladores do período de floração.

Florações seqüenciais das espécies de Psychotria e a semelhança de suas características florais (antese diurna, corola branca, tubulosa) e de hábitat (local sombreado do sub-bosque) parecem promover a "série de reposição" (sensu Macior 1971), ou seja, espécies distintas resultam, em conjunto, numa "única" floração, por um longo período durante o ano. Desse modo, há formação e retenção de uma "imagem de procura" alimentar por parte de polinizadores em comum (Thomson 1980), minimizando a competição por polinizadores entre as espécies. Estudos posteriores, sobre os visitantes florais dessas espécies, poderão confirmar essa hipótese.

As espécies estudadas, exceto $P$. hygrophiloides, apresentaram hercogamia recíproca, ou seja, as alturas dos estames e do estilete se equivalem nos morfos opostos (Ganders 1979, Barrett 1992) e, portanto, são distílicas. Entretanto, Castro \& Araújo (2004) comentaram que a hercogamia em $P$. nuda não foi exatamente recíproca, por apresentar uma grande sobreposição na altura dos estames e do estilete, entre os morfos florais. Em P. nuda, no presente estudo, não foi registrada esta sobreposição; convém salientar que o número de indivíduos amostrados foi menor que o dos referidos autores.

Segundo Pereira et al. (2006), P. hygrophiloides também é distílica, contudo apenas um dos morfos florais (flores brevistilas) foi registrado no local de estudo e, nos indivíduos amostrados, não foram observados frutos. A ausência de frutificação era esperada devido à auto-incompatibilidade e incompatibilidade intramorfo. $\mathrm{O}$ registro de apenas um dos morfos florais de P. hygrophiloides pode ser resultante da fragmentação da área de estudo. Esta espécie apresenta propagação vegetativa (observação pessoal), que parece ser uma estratégia alternativa de reprodução, comumente observada em espécies distílicas de Rubiaceae (Hamilton 1990, Rossi et al. 2005). Na ETPEAMP, observou-se um único agrupamento dessa espécie, que se vier a sofrer danos, pode acarretar a sua extinção local. Vale ressaltar, que $P$. hygrophiloides está pobremente representada em alguns dos maiores herbários do país (Pereira et al. 2006).

Além da diferença na altura dos estames e do estilete, as espécies estudadas apresentaram dimorfismo no comprimento da corola, dos lobos estigmáticos e das anteras. Embora Ganders (1979) tenha comentado que o dimorfismo no comprimento da corola pareça ser raro, essa característica tem sido observada em outras espécies de Rubiaceae: Rudgea jasminoides (Cham.) Müll. Arg. (Baker 1956), Palicourea fendleri Standl. e Palicourea petiolaris Kunth (Sobrevilla et al. 1983), Luculia gratissima (Wall.) Sweet (Murray 1990), Guettarda scabra (L.) Lam. (Richards \& Koptur 1993), Gaertnera vaginata Lam. (Pailler \& Thompson 1997), Palicourea padifolia (Willd. ex Roem. \& Schult.) C.M. Taylor \& Lorence (Ree 1997), Psychotria suerrensis Donn. Sm. (Faivre \& McDade 2001) e as espécies do presente estudo. Esses resultados indicam, ao contrário do mencionado por Ganders (1979), que diferenças no comprimento da corola podem ser comuns entre espécies distílicas. Em todas as espécies citadas, a corola foi maior na forma brevistila, exceto em $P$. suerrensis, na qual a forma longistila apresentou corola maior (Faivre \& McDade 2001). 

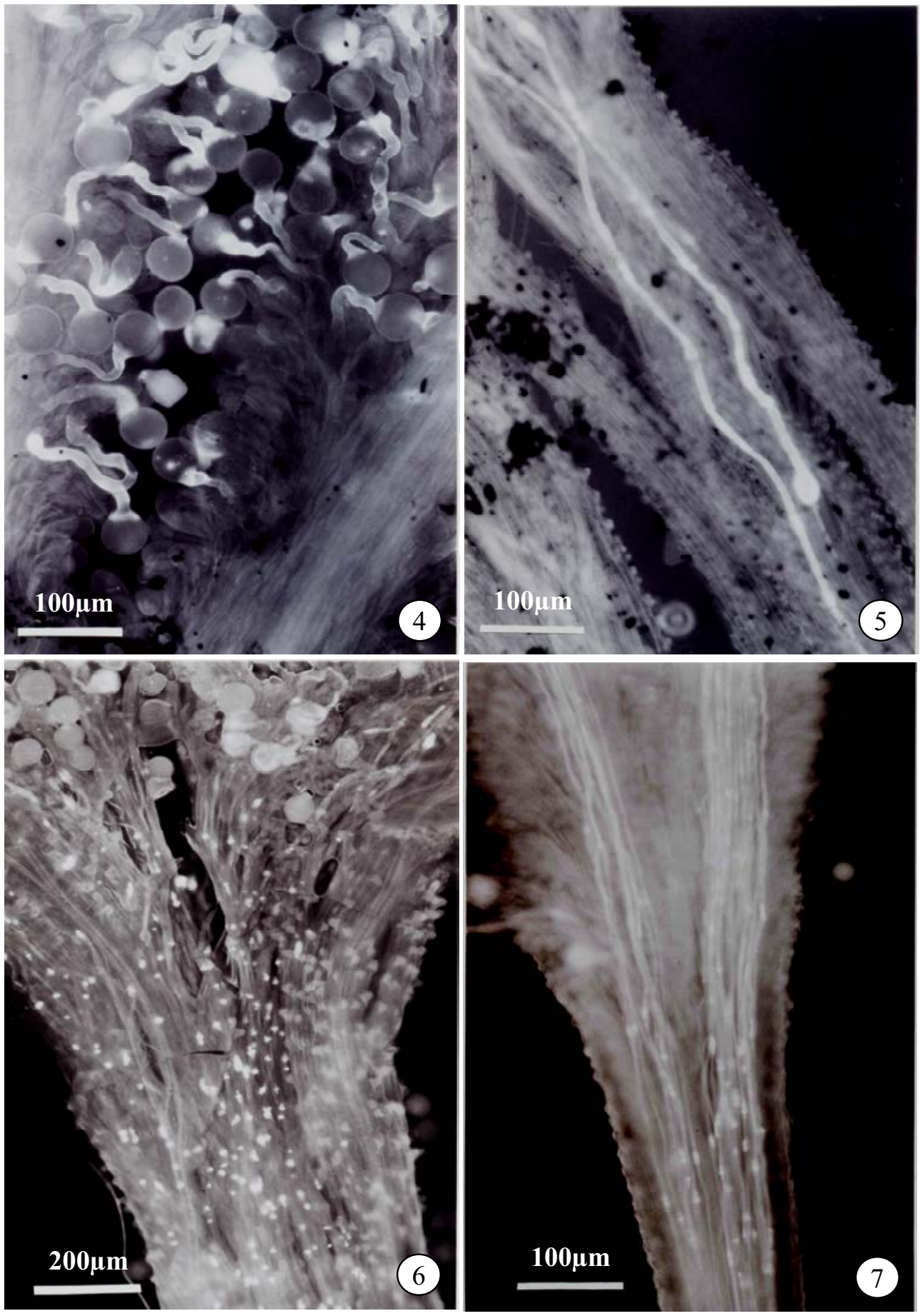

Figuras 4-7. Tubos polínicos resultantes de polinizações manuais em espécies distílicas de Rubiaceae, em Viçosa, Minas Gerais. 4. Psychotria nuda (Cham. \& Schltdl.) Wawra: polinização ilegítima (brevistila $\times$ brevistila), a inibição dos tubos polínicos ocorreu no estigma. 5. Palicourea longepedunculata Gardner: polinização ilegítima (longistila $\times$ longistila), a inibição de tubos polínicos ocorreu no estilete. 6. Rudgea lanceolata Nutt.: polinização legítima (brevistila $\times$ longistila), tubos polínicos no estigma e início do estilete. 7. Psychotria sessilis Vell.: polinização legítima, tubos polínicos, principalmente, no estilete.

Figures 4-7. Pollen tubes resulting from hand pollinations in distylous species of Rubiaceae, in Viçosa, Minas Gerais 4. Psychotria nuda (Cham. \& Schltdl.) Wawra: illegitimate pollination (thrum $\times$ thrum), inhibition of the pollen tubes in the stigma. 5. Palicourea longepedunculata Gardner: illegitimate pollination (pin $\times$ pin), inhibition of the pollen tubes in the style. 6 . Rudgea lanceolata Nutt.: legitimate pollination (thrum $\times$ pin), pollen tubes in the stigma and at the beginning of the style. 7 . Psychotria sessilis Vell.: legitimate pollination, pollen tubes in the style. 
Com relação ao comprimento dos lobos estigmáticos, Ganders (1979) comentou que as diferenças entre os morfos florais não são consistentes. De modo semelhante ao verificado para o comprimento da corola, têm sido demonstradas diferenças significativas entre as morfos florais, sob esse aspecto (Ornduff 1980, Sobrevilla et al. 1983, Murray 1990, Ree 1997, presente estudo).

Diferenças significativas no comprimento das anteras como encontradas em $P$. marcgravii e $P$. nuda também têm sido demonstradas por: Ree (1997) em Palicourea padifolia, Castro et al. (2004) em Psychotria birotula L. B.Sm. \& Downs, P. mapourioides DC. e P. nuda e por Rossi et al. (2005) em P. ipecacuanha (Brot.) Stokes. Anteras maiores em flores brevistilas, provavelmente, estão relacionadas ao tamanho do grão de pólen, que são, geralmente, maiores neste morfo floral (Teixeira \& Machado 2004a, Rossi et al. 2005). Entretanto, há necessidade de estudos posteriores, pois as espécies estudadas não tiveram seus grãos de pólen medidos.

Associado à hercogamia recíproca, as espécies distílicas, freqüentemente, ocorrem em populações isopléticas, ou seja, com razão equilibrada (1:1) entre os morfos florais na população (Ornduff 1971, Barrett 1992, Rossi et al. 2005), tal como observado na maioria das espécies aqui estudadas, exceto em $P$. marcgravii, $P$. conjugens e P. hygrophiloides. Neste contexto, essas espécies podem ser consideradas atipicamente distílicas, no local de estudo, por apresentarem populações anisopléticas. Segundo Barrett (1992), a anisopletia pode ser influenciada pela produção de novos indivíduos por propagação vegetativa e por haver formação de frutos decorrente de autopolinização. Entretanto, o desequilíbrio entre os morfos florais de $P$. conjugens e $P$. marcgravii não pode ser justificado com os argumentos de Barrett (1992), pois nenhuma dessas espécies, como observado em campo, se propaga vegetativamente e ambas são auto-incompatíveis. Novamente, a fragmentação pode ser o fator que favoreceu a quebra do equilíbrio entre os morfos. Por outro lado, esse desequilíbrio pode ser resultante de populações recémestabelecidas na EPTEAMP. Estudos posteriores, incluindo genética de populações, poderão esclarecer essa questão.

Em P. longepedunculata e $P$. hastisepala a inibição do crescimento dos tubos polínicos incompatíveis no estilete, em flores longistilas, assemelha-se ao registrado por Bawa \& Beach (1983) em Cephaelis elata Sw., Coussarea sp., Psychotria officinalis (Aubl.) Raeusch. ex Sandwith e Rudgea cornifolia (Knuth) Standl. e por Faivre (2002) em Psychotria chiapensis Standl. e P. poeppigiana Müll. Arg. Nas demais espécies estudadas, a inibição no estigma, em ambos os morfos florais, é comum e assemelha-se ao verificado em espécies distílicas de Rubiaceae (p. ex., Bawa \& Beach 1983, Murray 1990, Ree 1997).

Vários fatores parecem influenciar o crescimento de tubos polínicos e o local da rejeição de incompatibilidade. O tamanho do grão de pólen, que comumente varia entre morfos, poderia levar a diferentes ritmos de crescimento e locais de rejeição (Darwin 1877). As diferenças na morfologia e tamanhos das papilas estigmáticas entre morfos poderiam resultar em diferenças na germinação e crescimento dos tubos polínicos, que seriam afetados de maneira distinta pelas barreiras de incompatibilidade no gineceu (Lewis 1943, Shivanna et al. 1981). Fatores climáticos também parecem influenciar nas reações de incompatibilidade, evidenciando a complexidade destes mecanismos (Faivre 2002).

Em síntese, as espécies estudadas apresentaram o sistema de incompatibilidade esperado e parecem manter a reprodução sexuada (todas produziram frutos, exceto Psychotria hygrophiloides; Pereira et al. 2006), resultante de polinizações legítimas. Esse fato demonstra a atuação de polinizadores, apesar das espécies ocorrerem em fragmento florestal bastante alterado, local com fortes pressões seletivas interferindo na estabilidade das populações (Barret \& Richards 1990). Essas pressões poderão, no futuro, resultar em estados derivados como, por exemplo, autocompatibilidade e compatibilidade intramorfo, registrados em Psychotria acuminata Benth. (Bawa \& Beach 1983), Guettarda scabra (Richards \& Koptur 1993), Hedyotis salzmannii (DC.) Steud. (Riveros et al. 1995), Gaertnera vaginata (Pailler \& Thompson 1997) e Psychotria ipecacuanha (Rossi et al. 2005).

Agradecimentos - À coordenação de Aperfeiçoamento de Pessoal de Nível Superior (Capes) pela bolsa concedida à primeira autora; ao $\mathrm{CNPq}$ pela bolsa de produtividade concedida à segunda autora; aos funcionários do Herbário VIC e do laboratório de Anatomia Vegetal, do Departamento de Biologia Vegetal da UFV, pelo auxílio e presteza; à professora Luíza Sumiko Kinoshita pela leitura crítica do manuscrito e valiosas sugestões.

\section{Referências bibliográficas}

AIZEN, M.A. \& FEINSINGER, P. 1994. Forest fragmentation, pollination, and plant reproduction in a chaco dry forest, Argentina. Ecology 75:330-351.

ALMEIDA, E.M. \& ALVES, M.A. 2000. Fenologia de Psychotria nuda e P. brasiliensis (Rubiaceae) em uma 
área de floresta atlântica no sudeste do Brasil. Acta Botanica Brasílica 14:335-346.

BAKER, H.G. 1956. Pollen dimorphism in the Rubiaceae. Evolution 10:23-31.

BARRETT, S.C.H. 1992. Heterostylous genetic polymorphisms, model systems for evolutionary analysis. In Evolution and function of heterostyly (S.C.H Barret, ed.). Springer-Verlag, Berlim, p.1-29.

BARRETT, S.C.H. \& RICHARDS, J.H. 1990. Heterostyly in tropical plants. Memoirs of the New York Botanical Garden 55:35-61.

BARroso, G.M., PEIXOTO, A.L., COSTA, C.G., ICHASO, C.L.F., GUIMARÃES, F. \& LIMA, H.C. 1991. Sistemática de angiospermas do Brasil. $3^{\text {a }}$ ed., Universidade Federal de Viçosa, Viçosa.

BAWA, K.S. \& BEACH, J.H. 1983. Self-incompatibility systems in the Rubiaceae of a tropical lowland wet forest. American Journal of Botany 70:1281-1288.

BAWA, K.S., PERRY, D.R. \& BEACH, J.H. 1985. Reproductive biology of tropical lowland rain forest trees. I. Sexual systems and incompatibility mechanisms. American Journal of Botany 72:331-338.

CASTRO, C.C. \& ARAÚJO, A.C. 2004. Distyly and sequential pollinators of Psychotria nuda in the Atlantic rain forest, Brazil. Plant Systematics and Evolution 224:131-139.

CASTRO, C.C., OLIVEIRA, P.E.M. \& ALVES, M.C. 2004. Breeding system and floral morphometry of distylous Psychotria L. species in the Atlantic rain forest, SE Brazil. Plant Biology 6:1-6.

COELHO, C.P. \& BARBOSA, A.G. 2003. Biologia reprodutiva de Palicourea macrobotys Ruiz \& Pavon (Rubiaceae): um possível caso de homostilia no gênero Palicourea Aubl. Revista Brasileira de Botânica 26:403413.

CONSOLARO, H., SILVA, E.C.B. \& OLIVEIRA, P.E. 2005. Variação floral e biologia reprodutiva de Manettia cordifolia Mart. (Rubiaceae). Revista Brasileira de Botânica 28:85-94.

COSTA, M.L., ANDRADE, A.C.S. \& PEREIRA, T.S. 1997. Fenologia de espécies arbóreas em floresta montana na Reserva Ecológica de Macaé de Cima. In Serra de Macaé de Cima: diversidade florística e conservação em Mata Atlântica (H.C. Lima \& R.R. Guedes-Bruni, eds.). Jardim Botânico, Rio de Janeiro, p.167-186.

DARWIN, C. 1877. The different forms of flowers on plants of the same species. J. Murrary, London.

DULBERGER, R. 1992. Floral polymorphisms and their functional significance in the heterostylous syndrome. In Evolution and function of heterostyly (S.C.H. Barrett, ed.). Springer-Verlag, Berlim, p.41-84.

FAIVRE, A.E. 2002. Variation in pollen tube inhibition sites within and among three heterostylous species of Rubiaceae. International Journal of Plant Sciences 163:783-800.

FAIVRE, A.E. \& McDADE, L.A. 2001. Population-level variation in the expression of heterostyly in three species of Rubiaceae: does reciprocal placement of anthers and stigmas characterize heterostyly? American Journal of Botany 88:841-853.

GANDERS, F.R. 1979. The biology of heterostyly. New Zealand Journal of Botany 17:607-635.

GENTRY, A.H. \& EMMONS, L.H. 1987. Geographical variation in fertility, phenology and composition of the understory of Neotropical forests. Biotropica 19:216227.

GIBBS, P. 1990. Self-incompatibility in flowering plants: a neotropical perspective. Revista Brasileira de Botânica 13:125-136.

HAMILTON, C.W. 1990. Variations on a distylous theme in a Mesoamerican Psychotria subgenus Psychotria (Rubiaceae). Memoirs of the New York Botanical Garden 55:62-75.

HERMAN, B.P., TARUM, K.M., RUSSEL, J.W. \& DOLLAHON, N.R. 1999. Quantitative evaluation of stigma polymorphism in a tristylous weed, Lythrum salicari (Lythraceae). American Journal of Botany 86:1121-1129.

LEWIS, D. 1943. The physiology of incompatibility in plants. II. Linum grandiflorum. Annals of Botany $89: 157-63$

MACIOR, L.W. 1971. Coevolution of plants and animalssystematic insights from plant-insect interactions. Taxon 20:17-28.

MARTIN, F.W. 1959. Staining and observing pollen tubes in the style by means of fluorescence. Stain Technology 34:125-128.

MARTIN-GAJARDO, I.S. \& MORELLATO, L.P.C. 2003. Fenologia de Rubiaceae do sub-bosque em Floresta Atlântica no sudeste do Brasil. Revista Brasileira de Botânica 26:299-309.

MURRAY, B.G. 1990. Heterostyly and pollen-tube interactions in Luculia gratissima (Rubiaceae). Annals of Botany 65:691-698.

NETTANCOURT, D. 1977. Incompatibility in angiosperms. Springer Verlag, New York.

NEWSTRON, L.E., FRANKIE, G.W. \& BAKER, H.G. 1994. A new classification for plant phenology based on flowering patterns in lowland tropical rain forest trees at La Selva, Costa Rica. Biotropica 26:141-159.

ORNDUFF, R. 1971. The reproductive system of Jepsonia heterandra. Evolution 25:300-311.

ORNDUFF, R. 1980. Heterostyly, population composition and pollen flow in Hedyotis caerulea. American Journal of Botany 67:95-103.

PAILLER, T. \& THOMPSON, J.D. 1997. Distyly and variation in heteromorphic incompatibility in Gaertnera vaginata (Rubiaceae) endemic to La Reunion Island. American Journal of Botany 84:315-327. 
PEREIRA, Z.V., CARVALHO-OKANO, R.M. \& GARCIA, F.C.P. 2006. Rubiaceae Juss. da Reserva Florestal Mata do Paraíso, Viçosa, Minas Gerais, Brasil. Acta Botânica Brasílica 20: 207-224.

REE, R.H. 1997. Pollen flow, fecundity, and the adaptive significance of heterostyly in Palicourea padifolia (Rubiaceae). Biotropica 29:298-308.

RICHARDS, J.H. \& KOPTUR, S. 1993. Floral variation and distyly in Guettarda scabra (Rubiaceae). American Journal of Botany 80:31-40.

RIVEROS, G.M., BARRÍA, O.R. \& HUMAÑA, A.M. 1995. Self-compatibility in distylous Hedyotis salzmannii (Rubiaceae). Plant Systematics and Evolution 194:1-8.

RIZZINI, C.T. 1992. Tratado de fitogeografia do Brasil: aspectos ecológicos, sociológicos e florísticos. Âmbito Cultural, São Paulo.

ROBBRECHT, E. 1988. Tropical woody Rubiaceae. Opera Botanica Belgica 1:1-127.

ROSSI, A.A.B., OLIVEIRA, L.O. \& VIEIRA, M.F. 2005. Distyly and variation in floral traits in natural populations of Psychotria ipecacuanha (Brot.) Stokes (Rubiaceae). Revista Brasileira de Botânica 28:285-294.

SHIVANNA, K.R.J., HESLOP-HARRISON, J. \& HESLOPHARRISON, Y. 1981. Heterostyly in Primula. 2. Sites of pollen inhibition, and effects of pistil constituents on compatible and incompatible pollen tube growth. Protoplasma 107:319-337.

SOBREVILlA, C., RAMIREZ, N. \& ENRECH, X. 1983. Reproductive biology of Palicourea fendleri and P. petiolaris (Rubiaceae), heterostylous shrubs of a tropical cloud forest in Venezuela. Biotropica 15:161-169.

TEIXEIRA, L.A.G. \& MACHADO, I.C. 2004a. Sabicea cinerea Aubl. (Rubiaceae): distilia e polinização em um fragmento de floresta atlântica em Pernambuco, Nordeste do Brasil. Revista Brasileira de Botânica 27:193-204.

TEIXEIRA, L.A.G. \& MACHADO, I.C. 2004b. Biologia da polinização e sistema reprodutivo de Psychotria barbiflora DC. (Rubiaceae). Acta Botanica Brasilica 18:853-862.

THOMSON, J.D. 1980. Skewed flowering distributions and pollinator attraction. Ecology 61:72-579.

VELOSO, H.P., RANGEL FILHO, A.R. \& LIMA, J.C.A. 1991. Classificação da vegetação brasileira, adaptada a um sistema universal. IBGE, Rio de Janeiro.

VIANELLO, R.L. \& ALVEZ, A.R. 1991. Meteorologia básica e aplicações. Universidade Federal de Viçosa. Viçosa.

ZAR, J.H. 1999. Biostatiscal analysis. $4^{\text {th }}$ ed., Prentice Hall, Upper Saddle River. 